\title{
Symbols of a cosmic order
}

\author{
F. Hadi Madjid ${ }^{\mathrm{a}}$, John M. Myers ${ }^{\mathrm{b}, *}$ \\ a 82 Powers Road, Concord, MA 01742, USA \\ ${ }^{b}$ Harvard School of Engineering and Applied Sciences, Cambridge, MA 02138, USA
}

\begin{abstract}
The world runs on networks over which signals communicate sequences of symbols, e.g. numerals. Examining both engineered and natural communications networks reveals an unsuspected order that depends on contact with an unpredictable entity. This order has three roots. The first is a proof within quantum theory that no evidence can ever determine its explanation, so that an agent choosing an explanation must do so unpredictably. The second root is the showing that clocks that step computers do not "tell time" but serve as self-adjusting symbol-handling agents that regulate "logically synchronized" motion in response to unpredictable disturbances. Such a clock-agent has a certain independence as well as the capacity to communicate via unpredictable symbols with other clock-agents and to adjust its own tick rate in response to that communication. The third root is the noticing of unpredictable symbol exchange in natural systems, including the transmission of symbols found in molecular biology. We introduce a symbol-handling agent as a role played in some cases by a person, for example a physicist who chooses an explanation of given experimental outcomes, and in other cases by some other biological entity, and in still other cases by an inanimate device, such as a computer-based detector used in physical measurements. While we forbear to try to explain the propensity of agents at all levels from cells to civilizations to form and operate networks of logically synchronized symbol-handling agents, we point to this propensity as an overlooked cosmic order, an order structured by the unpredictability ensuing from the proof. Appreciating the cosmic order leads to a conception of agency that replaces volition by unpredictability and reconceives the notion of objectivity in a way that makes a place for agency in the world as described by physics. Some specific implications for physics are outlined.
\end{abstract}

Keywords: symbol, logical synchronization, idiosyncratic phase, symbol-handling agent, Turing machine, evidence vs. explanation.

\footnotetext{
*Corresponding author

Email addresses: gmadjideaol . com (F. Hadi Madjid), myers@seas . harvard.edu (John M. Myers)
} 


\section{Introduction}

Physicists find numerically expressed regularities in a world that every day surprises us all with its irregularities. Recently, commenting on Canales' book about Bergson and Einstein [1], Crease pointed to an "experiential amnesia" in physics [2], an amnesia that blocks attention to pre-conditions for physical time: "Bergson was trying to bring to light a sense of time presupposed in the construction of physical time itself-indeed, in Einsteins own effort to give to such time a definitive, mathematical formula." The thesis of the present report is that "time" as it works in physics is built out of networks of agent-clocks that do not dumbly tick, but that self-adjust in response to unpredictable communications from other clocks of the network. Examining both engineered and natural networks of clocks reveals an unsuspected order that depends on contact with an unpredictable entity. Attention to this unsuspected order has several implications, including an impact on the notion of scientific objectivity.

Our exposition of this "cosmic order" has three roots. The first root is the sharpening of a distinction obscured in today's theoretical physics, namely the distinction between obtaining numerically expressed evidence from experiments on the laboratory bench and explaining that evidence in mathematical symbols on the blackboard. As reviewed in Sec. 2, the sharpening of the distinction between physical numerical evidence and numbers calculated from a theory rests on a proof within the mathematics of quantum theory that no amount of evidence, represented in quantum theory in terms of probabilities, can uniquely determine its explanation in terms of wave functions and linear operators. Beyond mere opinion, the proof enables a clarity of thought otherwise unattainable in the distinction between measured and calculated numbers. The proof underpins all the work presented here. Building on the proof we show a heretofore overlooked unpredictability of explanations, an unpredictability beyond quantum uncertainty. The choice of an explanation requires an unpredictable reach beyond logic, a fact that challenges the traditionally notion of objectivity and that precludes any "final answers."

The second root stems from our experience with the design of clocking for fault-tolerant computer networks. A computer operates one step after another, regulated by the ticks of its clock. Fault tolerance is achieved by using a cluster of several computers, all designed to do the same task; each computer makes its computational moves in step with the others, and the computers compare notes at each step. Their clocks are organized in a network in which each clock regulates

its tick rate to stay close enough to the other functioning clocks for comparisons to 
make sense, but loosely enough so that if one clock fails, the other clocks continue. This requires self-adjusting clocks used not primarily to "tell time" but as agents that regulate motion. Such a clock-agent has a certain independence as well as the capacity to communicate with other clock-agents and to adjust its own tick rate in response to that communication.

Clocks-as-agents are required also by the national and international organizations that generate time broadcasts. As spelled out in [3], no two clocks, even those that "define" the international second as a unit of time, tick quite alike. For this reason, and because any single clock can fail, the time broadcasts generated by the National Institutes of Science and Technology (NIST) depend on several clocks linked by communicated symbols, each clock adjusting its tick rate so as to receive those symbols at a suitable phase. The self-adjustment of clocks requires both computation and response to unpredictable events, two capacities that might be called "cognitive". In this way our notion of clock-as-agent has come to differ rather dramatically from the popular image of "a clock." In Sec. 3 we discuss networks of symbol-handling agents equipped with such clocks, linked pairwise by communicated symbols, with each agent's clock adjusting its tick rate so as to receive those symbols at a suitable phase.

The third root is a direction for future research set by noticing unpredictable symbol exchange in natural systems, including the transmission of symbols found in molecular biology.

We think of a symbol-handling agent as a role played in some cases by a person, for example a physicist who chooses an explanation of given experimental outcomes or a person in a bucket brigade, and in other cases by some other biological entity, and in still other cases by an inanimate device, such as a computerbased detector used in physical measurements. We think of a symbol-handling agent as exhibiting three capabilities:

1. an agent can transmit and receive symbol-carrying signals to and from other agents;

2. an agent can transmit symbols that cannot be predicted prior to their transmission;

3. circumstances permitting, an agent maintains a form of synchronizationto be called logical synchronization-with one or more other agents, which requires that an agent manage the tick rate of its clock.

We will speak of anything that exhibits these three capabilities as a symbol-handling agent, or sometimes, for short, just as an agent. (In earlier work we spoke of symbol-handling agents as live clocks [4] or open machines [5]) 
As a descriptive form, a network of symbol-handling agents is broad enough to encompass the computer network that mediates communications among physicists, e.g. the internet with its traffic in both experimental and theoretical matters, and also as a form that can be applied to describe naturally arising symbolic communication, for example as in the molecular signals of biology. Below we discuss the communication of symbols among agents as a neglected topic of physics.

To model some of the physical behavior of symbol-handling agents linked in communications networks, we widen an approach pioneered by Turing. Turing introduced the Turing machine to model a facet of human cognitive capacity. In school we all learn to do sums, to multiply and divide, in short, to compute-a word that in 1936 meant a capability primarily thought of as a human capability. In that year Turing abstracted that particular cognitive capability to produce a specification for an inanimate physical machine. The Church-Turing thesis states that a function on the natural numbers is computable by a human being following an algorithm, ignoring resource limitations, if and only if it is computable by a Turing machine [6]. Since 'what is computable by a human being' is an informal notion, it has no formal definition, so the thesis, although it has near-universal acceptance, cannot be formally proven.

In essence, the agent role that we model is that of a physical sequential processor, that is, a processor that takes one step after another, with the "next step" influenced not just by a stored program but also by momentary contact with an unpredictable entity. (Positing that an agent works sequentially answers the question: what is the difference between one agent and two agents? Two agents can do two things concurrently, but one agent can't.) Our "agent as a physical sequential processor" consists of a self-adjusting clock that regulates the motion of a Turing machine which is modified to allow its contact with an unpredictable entity. For this we use not the usual Turing machine but Turing's Choice Machine (which we capitalize for emphasis). The Choice Machine has the feature, crucial to our model of an agent, that it can receive symbols from an unpredictable "outside". Note that the Choice Machine, unlike most modern practice in computer science, enforces no separation between program and "data", so that symbols received from the unpredictable "outside" can work as programs.

Our model of an agent opens a crack in the traditional physicalist image of clockwork as an explanatory principle in two ways:

1. By invoking Turing's Choice Machine, we put the 'physical sequential processor' in contact with an unpredictable entity; and

2. We reformulate the concept of a clock to account for how clocks actu- 
ally work in modern technical contexts, involving communication between clocks and their rate adjustments in response to unpredictable measured phases.

Years before developing the proof that separates evidence from its explanations, we experimented on the laboratory bench with conditions under which the interleaving of two sequences of symbols fails. As described in Sec. 4, these experiments on instabilities in a decision-making device (a flip-flop) clarified the circumstances under which two agents could be expected to agree about the symbol presented to them at a shared moment. The experimental results on agreement and disagreement contribute to a re-definition of the notion of "objectivity." In addition they open an avenue of what might be called "the physics of borderline cases."

Section 5 discusses symbol-handling agents in physics as they appear in several contexts:

1. The experimental and theoretical working of physicists is mirrored in the memories - think Turing tapes — of the computerized agents that mediate their communications, e.g. over the internet.

2. Networks of symbol-handling agents, many of them automated, serve as tools for experimental inquiry, especially in cases where extreme precision is needed, as in the Laser Interferometer Gravitational Observatory (LIGO).

3. Networks of symbol-handling agents are promising as metaphors by which to describe physical activity, for example in biophysics.

It will be shown that the need for logical synchronization impacts all these contexts; in particular, in situations requiring the highest attainable precision of motion, networks of symbol-handling agents cannot derive their timing as users of national time broadcasts; they have to build their own "time."

To reach our conclusions we make the following three assumptions:

1. We assume we can extend the proposition proven in quantum theory concerning the unpredictability of explanations of given evidence to physics in general.

2. We partition the (limited) cognitive capabilities of agent that we need to be concerned with into computation on one side and contact with an unpredictable entity on the other.

3. We assume the Church-Turing thesis. 
4. We assume that the communication of sequences of symbols among agents, including people, is physical, not in the physics of a clockwork automaton, but rather in the physics that we are trying to explicate, a physics that has room in it for irreducible unpredictability beyond quantum uncertainty. In particular, we assume that one agent, say Agent A, in contact with an unpredictable device such as a photo-detector, can obtain from that device a number that a second Agent B, human or not, can find out only by the transmission of the number from agent $\mathrm{A}$ to $\mathrm{B}$ by a signal carrying numeric symbols that express the number.

\section{Remarks:}

1. We note that the third and fourth assumptions may not be to everyone's taste; for alternative, non-reductive views, see [7, Sec. 5.3].

2. We do not offer a theory of cognition; rather we leave most of the many capabilities that lurk under the umbrella term cognition undiscussed; we deal only with two: the capability to compute, whether by hand or by machine, and the capability to guess an explanation, which, as outlined in Sec. 2, requires an unpredictable act of a person in contact with an unpredictable entity, provably necessary to physics. We focus on these two cognitive capabilities to get at the role of unpredictability heretofore overlooked in physics. Nor do we by any means offer a complete characterization of "agency", but rather a beachhead into a restricted class of symbol-handling agents as descriptive elements that have been demonstrated to have interesting applications. In particular we avoid attaching to agency any notion of volition; we escape the need to do so by putting in its place an agent's responsiveness to something unpredictable.

3. We put a big emphasis on an agent's capacity to issue unpredictable numeric symbols, and one may ask "to what do we ascribe the issuance by an agent of numeric expressions that are unpredictable?" This is essentially a question of philosophy or religion, any answer to which must venture beyond science. We like to think of the agent issuing such an expression as being in contact with an unknowable entity, to which, within the bounds of science, we are precluded from ascribing any additional features.

While we forbear to try to explain the abundance of behavior at all levels from cells to civilizations aptly describable as consisting of logically-synchronized networks of symbol-handling agents in contact with something unpredictable, in the discussion in Sec. 6 we will point to this abundance as an overlooked cosmic order. 


\section{Unpredictability stemming from logically undefined choices in explaining given evidence}

We begin this section with a reminder that several varieties of unpredictability are endemic to physics. One familiar kind of unpredictability is the uncertainty that pertains to a spread in a probability measure over a given set of possible outcomes, implied by quantum theory for a generic measurement. Uncertainty makes the outcome at any particular occurrence of a measurement unpredictable, and in situations that require prompt action, discussed in Sec. 5, this unpredictability matters. Another kind of unpredictability is implied by Gödel's 1931 proof of the existence of undecidable propositions in arithmetic, and still another kind by Turing's 1936 proof of the existence of uncomputable functions. Until fairly recently one might suppose that uncomputable functions were to be found only in areas of number theory remote from physics, but now examples are known of undecidable functions within quantum theory. Furthermore, as we shall see, Turing's characterization of computation advanced not just mathematics, but also physics.

In connection with Turing computability, we note the distinction between defining a function and computing its values. For example, the definition of the square root 2 is distinct from the act of calculating some number of places of the square root of 2 as a decimal expression. The distinction between defining and computing allows one to speak of certain choices as not merely uncomputable but as logically undefinable. Our prime example is the choice of an explanation of given evidence, as expressed within quantum theory. We now review how this choice of an explanation provably requires a reach beyond logic.

Quantum theory serves as a mathematical language by which to think about experiments. Quantum language is built on a skeleton of a Hilbert space of states and the Born trace rule. This skeleton imparts a mathematical form to quantum language that remains stable while hypotheses with physical content come and go. From this skeleton alone, we proved in [8] that explanations cannot be determined uniquely from evidence.

The proof can be stated in simplified form, as follows. To recall the Born trace rule, we express quantum states as density operators, and we express measurements by positive operator-valued measures (POVMs). The simplest POVMs, which are all that we need for a simplified statement of the proof, assume a finite or a countably infinite set of outcomes and associate a positive operator $M_{i}$ to the $i$-th outcome. We call these $M_{i}$ measurement operators. (They sum to the identity operator: $\sum_{i} M_{i}=1$.) In explaining an experiment in quantum language, one views the experiment as consisting of a number of trials, each of which involves 
a preparation followed by a measurement. One represents the preparation by a density operator that can vary from trial to trial. One expresses the measurement by a POVM that can also vary from trial to trial. Given a density operator $\rho$ and a measurement operator $M_{i}$, the probability of outcome $i$ is given by the Born trace rule as

$$
\operatorname{tr}\left(\rho M_{i}\right)=\operatorname{Pr}(\text { outcome i) }
$$

The Born trace rule expressed in (1) relates probabilities on the right side of the equation to a density operator and a measurement operator on the left side of the equation. An experiment generates outcomes that can be tallied to approximate probabilities, and the probability on the right side represents theoretically, on the blackboard, so to speak, the most one can hope for in the way of numerical evidence from an experiment. On the left side of the equation, the density operator and the measurement operator express an explanation of the evidence. Going from left to right in Eq. (1), given a density operator and a measurement operator, the Born trace rule tells how to calculate a unique probability; many textbook problems ask for just such calculations. But there are no operators to be seen on the bench, so what happens when an experiment shows something unpredicted and a new explanation is needed? This question presents the inverse problem: given the experimental outcomes interpreted as probabilities on the right side of the equation, one seeks a combination of a density operator and a set of measurement operators that generates the given probabilities. While the language of quantum theory makes available density operators and measurement operators as terms to write on the blackboard, it cannot tell you which density operators and measurement operators to write in order to explain any particular experiment: for this inverse problem there is no unique solution. Given any probability measure on a countable set of outcomes, we proved that there are infinitely many POVMs and density operators that generate the given probability measure. Thus whatever experimental evidence is on hand admits of numberless explanations. Choosing one or indeed any finite number of these explanations requires a reach beyond logic; one has to guess [8]. And a physicist's guessed explanation is logically unpredictable.

Any experiment can be extended, e.g. by the insertion of another light source, another detector, another filter, etc. Correspondingly, any explanation can be viewed as a restriction to a special case of an explanation of an extended experiment [9]. The numberless explanations that generate a probability measure over a given parameter domain disagree among themselves with respect to the extensions from which they can be restricted. This disagreement implies that ex- 
planations are almost certain to require revision when tested over enough of their extensions. The picture emerges of an open cycle of the making of guessed explanations, testing their extensions, and revising the guesses; that is, guessing again.

\section{Remarks}

1. The conflicts among predictions of extended explanations are not limited to small differences associated with issues of precision. In some cases, two explanations that generate given probabilities have drastically different implications. An example concerns quantum key distribution, for which two explanations fit a given probability measure, but one explanation asserts that the quantum key distribution is secure against undetected eavesdropping while the other explanation asserts that the key distribution is totally insecure [10].

2. To make a hypothesis in physics is to reach beyond logic to assert that a mathematical structure represents certain physical behavior. Because of its inescapable dependence on guesswork, the hypothesis is subject to eventual falsification. It is worth noting that, unlike a hypothesis stated in the language of quantum theory, the language itself is not falsifiable: given any probability measure on a countable set of outcomes, there exist (many) quantum explanations, so there can be no probabilities that lie outside the reach of quantum language [10]. Being unfalsifiable, the language of quantum theory offers relatively stable mathematics. The proof of the need for guesswork to choose an explanation is not a hypothesis of physics, but a proof within mathematics.

3. Coming up with an explanation necessarily involves an act of imagination beyond anything calculable. An act of imagination requires intimate contact with something unknowable. (If you know it, you don't have to imagine it.) The finding that explanations cannot be calculated from data but require contact with something unknowable has the following implication that adds to the necessity in physics for communication. An person's act of imagination expressed in symbols remains unknowable to a second person unless the symbols of the expression are communicated from the person to the second person.

4. Recognizing guesswork and its vulnerability to occasions for revision as indispensable to explanations precludes the possibility of assuring any final answer or any "objective truth." Yet, under circumstances to be discussed in the next section, agents can communicate sequences of numerals expressing 
evidence and explanations, numerals about which they can be expected to agree; remarkable too is that the actions needed to maintain the communications channels depend on idiosyncratic contact with something unknowable, beyond collective agreement.

\section{Networks symbol-handling agents}

This section is dense with detail needed for the design of experiments aimed at exploring the cosmic order. In it we give modeling language to express agents that recognize symbols carried by signals. By symbols, we mean elements of communication among agents, human or not, that can lead to action involving energy supplied not by the symbols themselves nor by the agent that sends them but by the receiving agent. How a receiving agent responds to a symbol depends on the past history of the agent. Symbols can be conveyed from agent to agent in various ways, for example by: (1) letters, words, and numerals, expressed as written characters, and (2) electronic impulses carrying bits in a computer, and (3) molecules involved in biological signaling. Symbols used by people convey elements of thought, often prompting actions and emotions. And symbols convey the calculational traffic to be found both in man-made computers and in the biological processes of living creatures.

Whether to say signal or symbol as we use these words is a matter of point of view, and not a matter of distinguishing two distinct things. Consider a pawn on a chess board. From the viewpoint of players, the pawn is a symbol. A player is indifferent to variations within certain bounds of its shape, its weight, and when and how it is placed on a square of the chessboard, e.g. whether it is a little off center doesn't matter. For the craftsman who makes the pawn, however, its signal aspect matters: its shape, its weight, the material of which it is made. The same holds for the 0 's and 1's in a computer. To a programmer they are symbols, while to an electrical circuit designer the details of the physical signals that the programmer views as symbols matter greatly. It is a fact, indeed to us an amazing fact, that symbols embodied by signals with large tolerances can take part in mechanisms that act with tight tolerances. This is what happens in computer-controlled machining: the signals that carry the $0 \mathrm{~s}$ and $1 \mathrm{~s}$ in the computer can vary within broad limits without interfering with the mathematical exactitude of the calculations that control the shapes that are machined.

Numeric symbols arrive one after another, and the sequence matters: "1011" is a different message from "1110". An agent must deal with symbols in a way that respects the order in which they arrive, making it convenient to view an agent 
as a sequential processor. Turing broke new ground by expressing a sequential processor mathematically by his "machines", both what today is called a Turing machine and a lesser known Choice Machine that plays the crucial role in our model of an agent.

Offering mathematical structure to represent physical behavior is the business of physics, and in his "machines," Turing made an interpretive leap beyond the reach of mathematics to create novel physics. In representing the physical activity of computing, he did not limit himself the terms (e.g. "particles and fields") expected in physics; rather, he introduced new terms (e.g 'tape', 'scanned square', 'moment', 'move') peculiarly appropriate to describing the activity of a calculational agent. In retrospect, one also sees physics implicit in Gödel's 1931 proof of undecidability, because proving a proposition is a physical activity: one has to write into a recording medium, and in checking a claimed proof, one reads the written record.

In the rest of this section we consider agents who communicate by means of transmitted sequences of symbols from one agent to another. We aim to show the critical role of the timing required. To this end we assume that each agent is a sequential processor. As a model of an agent that communicates with others, we start with a Turing machine as a model of a sequential processor, and then modify it, first by giving the machine the capacity to transmit and to receive symbols from other such machines, and secondly by modifying the Turing machine to be stepped by an accompanying clock that can have its rate adjusted by commands from the Turing machine.

The Turing machine as a sequential processor is elegantly described in Turing's 1936 paper:

We may compare a man in the process of computing a real number to a machine which is only capable of a finite number of conditions $q_{1}, q_{2}, \ldots, q_{R}$ which will be called " $m$-configurations". The machine is supplied with a "tape" (the analogue of paper) running through it, and divided into sections (called "squares") each capable of bearing a "symbol". At any moment there is just one square, say the $r$-th, bearing the symbol $S(r)$ which is "in the machine". We may call this square the "scanned square". The symbol on the scanned square may be called the "scanned symbol". The "scanned symbol" is the only one of which the machine is, so to speak, "directly aware". However, by altering its $m$-configuration the machine can effectively remember some of the symbols which it has "seen" (scanned) previously. The 
possible behaviour of the machine at any moment is determined by the $m$-configuration $q_{n}$ and the scanned symbol $S(r)$. This pair $q_{n}$, $S(r)$ will be called the configuration: thus the configuration determines the possible behaviour of the machine. In some of the configurations in which the scanned square is blank (i.e. bears no symbol) the machine writes down a new symbol on the scanned square: in other configurations it erases the scanned symbol. The machine may also change the square which is being scanned, but only by shifting it one place to right or left. In addition to any of these operations the $m$-configuration may be changed. Some of the symbols written down will form the sequence of figures which is the decimal of the real number which is being computed. The others are just rough notes to "assist the memory". It will only be these rough notes which will be liable to erasure.[11]

In a side remark in the same paper, Turing briefly introduced an alternative machine called a choice machine, contrasted with the usual Turing machine that Turing called an a-machine:

If at each stage the motion of a machine ... is completely determined by the configuration, we shall call the machine an "automatic machine" (or a-machine). For some purposes we might use machines (choice machines or c-machines) whose motion is only partially determined by the configuration .... When such a machine reaches one of these ambiguous configurations, it cannot go on until some arbitrary choice has been made by an external operator. This would be the case if we were using machines to deal with axiomatic systems [11].

One of the two ingredients in our model of a symbol-handling agent is a Choice Machine modified so that it can take part in a communications network by transmitting symbols to other such machines and, under certain conditions of "logical synchronization," can receive symbols transmitted to it by other machines. To assure the unpredictability of a symbol-handling agent, we posit that an "external operator" chooses a symbol and writes it onto the scanned square of the agent's Choice Machine privately, in the sense that the symbol remains unknown to other agents unless and until the symbol-handling agent that receives the chosen symbol reports it to others. 


\subsection{The motion of symbol-handling agents.}

Sequences of symbols on the squares of a tape at a moment are static-they show no motion. To deal with the physics of motion, one needs to deal not just with recorded sequences but also with temporal sequences, such as sheep herded one after another through a gate or the back-and-forth swings of a pendulum.

To express motion mathematically, one faces the hurdle that what a mathematical function does is to assign an element of a codomain to each element of a domain, with both the domain and the codomain thought of as static. From the standpoint of physics, motion expressed mathematically is essentially motion captured in the frames of a movie film detached from information about scale and speed. The movie film would be the same if the scene filmed were sped up by a factor and the clock that drives the movie camera were sped up by the same factor, so that in mathematics one cannot express motion per se. In preparation for discussing the regulation of the motion of agents necessary to their exchange of symbols, we flag two points:

1. In the mathematics of the Choice Machine, a 'move' is a mapping of a machine configuration at one moment to a machine configuration at the next moment, and a sequence of moments is like a sequence of frames of a movie film, which, by itself, contains no specification of its physical motion, for example, how rapidly it is to be moved.

2. Implicit in the interpretation of the Choice Machine as representing the physical action of computation is the subdivision of the moment into a phase in which the machine can read the scanned square, so that its next action is determined, and a distinct other phase in which the machine can write on the square. This subdivision is necessary to avoid a conflict between trying to read and trying to write on the same square at once.

With these points in mind, we think of a Choice Machine moved by the ticks of a clock at a rate adjustable (relative to the clock's own unadjusted internal standard) by commands from the Turing machine itself, as discussed in [4, 5]. If we picture the clock as having a single hand that cycles around a dial, then subdivisions of the dial correspond to phases of the computational cycle of the Turing machine, with a phase in which a symbol can be written on the scanned square. This modified Choice Machine expresses a computer that can take part in a communications network, and as such combines both the logic and the motion required of a process-control computer in contact with an unpredictable environment. The modified Choice Machine stepped by its adjustable ticks models our symbol-handling agent. 


\subsection{Logical synchronization.}

We call the condition in which symbols arrive during a phase of the computational cycle in which they can be written into memory logical synchronization. The need for logical synchronization, long known to engineers of digital communications [12, 13], is reminiscent of a game of catch, in which a player cycles through phases of throwing and catching a ball, or perhaps more simply a spoken dialog in which each person alternates between listening and speaking. The requirement for logical synchronization constrains number-carrying networks. (If a spacetime manifold in invoked, logical synchronization put "stripes" on spacetime [5].)

Another image of logically synchronized symbol-handling is a bucket brigade, in which people work in a line, each passing a full bucket to the person to the right while receiving an empty bucket in exchange, which is next passed to the person to the left. The people in a bucket brigade work in dovetailing cycles. Each cycle contains a phase in which one person passes a full bucket to a neighboring person, sharing a rhythm: if you are to my right, then when you turn to the left, I turn to the right to pass my full bucket to you and to receive your empty bucket. If I try to pass you my full bucket outside of the phase in which you can receive it, we spill the bucket.

Logical synchronization requires more or less continual adjustment of clock rates to limit the drift of physical clocks. Unavoidable drift in clock rates stems from quantum uncertainty, from relative motion of the agents, and from other causes [5]. The adjustment of tick rates of agents' clocks entails feedback that responds to the phases at which transmitted symbols arrive. On the blackboard, we represent the cycle of the agent's clock by a unit interval of the readings of its adjustable clock, and we express a reading of a the clock as $m . \phi_{m}$ where an integer $m$ indicates a count of cycles and $\phi_{m}$ is the phase within the cycle. Choosing the convention that $-1 / 2<\phi_{m} \leq 1 / 2$, we model the phase of writing at which an agent can receive a character as corresponding to

$$
|\phi|<(1-\eta) / 2
$$

where $\eta$ (with $0<\eta<1$ ) is a phase interval that makes room for reading. When this phase constraint is met for a channel between a transmitting agent and a receiving agent, we say the receiving agent is logically synchronized to the transmitting agent.

The adjustment of the rate of an agent's clock in order to maintain logical synchronization with another agent proceeds as a balancing operation. We think 
of an agent's clock as having a "faster-slower lever." The "faster-slower lever" works like the pointer of a balance instrument that moves one way or the other in response to opposing impulses from an arriving symbol and from the agent's reference signal in the middle of the receptive phase. If the symbol arrives in the middle of the receptive phase, neither well before nor well after the reference signal, the response of the balance is indeterminate; it can tip a little either way or hover in the middle. While symbol recognition is invariant under limited variations in timing, making it also invariant under interchange of manufactured instruments, the balancing that drives rate adjustment will not be the same if the balancing instrument is interchanged with another of the same manufacture. We speak of the behavior of measured phases that vary when two balancing components of the same design are interchanged as idiosyncratic.

Logical synchronization depends on the happy fact that the idiosyncrasy endemic to balancing does not matter, because it occurs only when the symbol arrives more or less in the middle of the receptive phase, so that a small adjustment of clock rate cannot cause the next few symbols to arrive outside their receptive phases. If the agent's clock with its ambiguous small adjustments drifts enough so that the subsequent arrivals are noticeably early or late relative to the mid-phase aiming point, the operation of the balance becomes definite and adequately corrective.

Remark: Maintaining logical synchronization depends on prompt steering in response to deviations that can be expressed numerically only later, after they have been responded to. Furthermore, the numerical expression of the deviations is necessarily idiosyncratic in that neither two persons nor two machines can be expected to produce numerical records of phases that agree.

To represent the transmission of numerals from one agent to another on the blackboard, we follow Shannon in speaking of a communications channel; however we augment his information-theoretic concept of a channel [14] with the agent's clock readings at the transmission and reception of symbol-bearing signals [5]. We indicate the timing in a channel from agent $A$ to agent $B$, by a set of pairs, each pair of the form $\left(m . \phi_{m}, n . \phi_{n}\right)$. The first member $m . \phi_{m}$ is an $A$-reading at which agent $A$ transmits a signal and the second member $n . \phi_{n}$ is a $B$-reading at which agent $B$ registers the reception of the signal. In this way the notion of a channel is expanded to include the clock readings that indicate phases of signal arrivals that have to be controlled in order for the logical synchronization of the channel to be maintained. 
Proposition: A symbol can propagate from one agent to another only if the symbol arrives within the writing phase of the receiving agent.

Corollary: Exact agreement concerning symbols depends on idiosyncratic management of phases.

\section{Sequencing failures and the concept of objectivity}

What happens when a symbol-carrying signal arrives at a receiving agent just too late for the agent's writing phase? Forty years ago we investigated this question experimentally by examining the behavior of an elemental decision agent, a clocked flip-flop. Packed by the million on the silicon chips of communicating computers, the clocked flip-flop is a memory device into which a 0 or 1 can be written, provided that the symbol arrives during a phase in which the clock opens a gate. A flip-flop is the electronic analog of a hinge that records a 1 if flipped one way or a 0 if flopped the other way. In the case of an electronic flip-flop, an arriving 1 comes embodied as an electrical pulse of energy above a high threshold, and an arriving 0 comes with an energy below a low threshold, well under the high threshold. For a flip-flop set at 0 , a 1 arriving while the gate opens on a phase of writing can flip the hinge over to record a 1. In effect, the clocked flip-flop is aware of the possible symbols that it might receive, in that it balances any arriving signal against a reference energy level. If the pulse energy is above the reference level, the flip-flop both augments the pulse energy to lift it above the high threshold and flips the hinge over. If the pulse energy is below the reference level, the flip-flop drains its energy below the lower threshold and stays flopped back to indicate a 0 .

A symbol arriving after the clock closes the gate is shut out and ignored by the flip-flop; however, if a pulse of electrical energy conveying a 1 arrives just as the writing phase is ending, in a race with the clock's closing of the gate, the pulse squeaks through the closing gate into the receiving flip-flop as a "runt pulse." Then the hinge might flip to a 1 or might stay at 0 , but there is another possibilityindeed a possibility outside the frame in which digital signals are conventionally discussed: the runt pulse can be so close to the reference level that it lifts the hinge part way but not all the way over, leaving the hinge hung up in an unstable "in between" state, teetering on edge, until, eventually, it flips or flops [15, 16, 17].

This "in between" state occurs occasionally when a computer responds to unsynchronized input signals, and it leads to logical confusion, as follows. Computations require that a receiving clocked flip-flop $A$ transmit copies of its record not just to one following flip-flop but through a fan-out to a pair of flip-flops, say $B$ 
and $C$, so that whatever symbol was in $A$ at an earlier moment appears in both $B$ and $C$ at the next moment; that is, $B$ and $C$ both receive 0 or both receive 1 . But if flip-flop $A$ teeters in an unstable equilibrium, then flip-flops $B$ and $C$ may not only hang up, but can "fall differently" so that the symbol in $B$, instead of matching that in $C$, conflicts with it, which is what we mean by "logical confusion."

We explored the teetering of a flip-flop not just analytically on the blackboard, but as it takes place physically on an electronics bench. The results, reported in [8], were clear enough, but we could not describe what was to us the most interesting aspects of the experiment; words failed. With our subsequent proof that puts unpredictability squarely within physics, however, we can now tell the story. In particular, the experimental design to be described illustrates how the investigators and the laboratory bench shape each other, how the investigators must be aware of the need to adjust the devices on the bench, how the devices must be designed to be receptive to adjustment by the investigator, how unpredictable outcomes propagate from the bench to the investigator, and how the unpredictable responses of the investigator to these outcomes propagate back to the bench.

Our experiment to measure the teetering of a clocked flip-flop consisted of several billion trials of sending a 1-pulse to a clocked flip flop $A$ that, after an adjustable delay $T$, was read by two flip-flops $B$ and $C$. It took about 300 nanoseconds per trial. For each trial we arranged for the 1-pulse to race a gate-closing clock pulse, causing a runt pulse that made the flip-flop $A$ teeter on edge. To tell if $A$ was teetering on edge, we arranged the electronics to keep a running average over past trials to record how often the flip-flop, after teetering, fell to 1 vs. how often it fell to zero. If the running average was about even between 1's to 0's, then the timing of the of the 1-pulse relative to the clock pulse was likely to make the flip-flop teeter.

But when we first tried to set the timing so as to bring about this desired even running average, we ran into trouble. We generated both the clock pulse and the 1-pulse repetitively at $300 \mathrm{~ns}$ intervals through a delay line from a common signal generator, and we regulated the timing of the 1-pulse relative to the clock pulse by adjusting the delay of the delay line for the 1-pulse. The trouble was that however we adjusted the delay line, we got either all 1's or all 0's from the running average. Because of drift in the delay line and perhaps other unanalyzed effects, we could not get the very delicate balancing of the "hinge" that we wanted. The solution was to use feedback. We electronically read the running average and fed that back to automatically adjust the delay line. Feedback worked like a charm to put the flip-flop on edge well enough to run the experiment.

Another interesting feature of the experimental design was the detection of 
teetering. Prior investigations of teetering in flip-flops made use of oscilloscopes that operated on faster time scales than that of the flip-flop; in effect the oscilloscope acts as a movie camera to photograph the teetering of the (electronic) hinge. Instead of using an external high-speed "camera", we wanted an experimental design that works even if the flip-flop operates faster than any such external "camera." To this end, instead of an oscilloscope, we used the logical confusion ensuing from a runt pulse as a means of measurement, by using the pair of flipflops $B$ and $C$, as described above. We could then plot the statistics of how often $B$ and $C$ disagreed with each other as a function of the waiting time $T$, as reported in [8].

Feedback, though it worked like a charm, posed what then seemed to us to be a conundrum. We were used to thinking of an experiment as something that an investigator may start, but must then keep his or her hands off until a result emerges. With feedback, we put ourselves, or at least the automated feedback loop that acted for us, right into the operation of each trial, so that we were using outcomes from earlier trials to direct a current trial. Now we say: we as investigators act like symbol-handling agents, and that's what symbol-handling agents do. But still, the acceptance of feedback invites one to rethink the conventional devotion to "objectivity" as a Cartesian preclusion of the observer from tinkering with the observed. By admitting feedback from previous trials, one can explore unstable physical behavior not otherwise susceptible to investigation.

In connection with "objectivity" there is something interesting about the use the pair of receiving flip-flops $B$ and $C$ to detect teetering. When flip-flop $A$ is receiving a 1-symbol under conditions of logical synchronization, so the 1-symbol arrives well within the phase of writing, there is no disagreement between $B$ and $C$. The flip-flops $B$ and $C$ act as agents that are interchangeable. Such interchangeability is a hallmark of what we view as a reconceived "objectivity": over a sequence of trials in which $A$ is sent at one moment sometimes a 0 and sometimes a 1 , at the following moment $B$ and $C$ reliably show the same outcome: both show 0 or both show 1 . The discrepancies registered in the experiment show that the measurement of a phase $\phi$ cannot be objective in the way we mean it: two detecting agents can disagree; indeed we used what might be called the idiosyncrasy of the agents $B$ and $C$ to indicate the phase corresponding to the closing of the gate. As we said in the previous section, measurements of phase, necessarily idiosyncratic, are indispensable to the logical synchronization that enables interchangeable agents to agree about counts of cycles and sequences of symbols received.

In summary, by introducing a distinction between symbols registered inter- 
changeably by several agents and the idiosyncratic phases that must be responded to in order to maintain logical synchronization, the investigation of a race between a symbol and a clock tick exhibits a crack in the Cartesian veneer of disembodied objectivity in which theoretical physics pretends to wrap we who investigate:

1. The race condition could not be passively observed but had to be actively maintained in the face of disruptive effects that we could not predict.

2. Maintenance of the race condition depends on the investigator choosing a control algorithm that specifies how a feedback loop responds to unpredicted effects, a choice that requires an act of imagination.

3. The most sensitive indicator of a race condition is a "measurable breakdown in Cartesian objectivity": two detectors disagree.

\section{Unpredictable, symbol-handling agents in physics}

\subsection{Physicists communicate with each other and with their experimental devices.}

Physics depends on the transmission of symbols, such as the sequences of symbols that make numerical records, both records of experimental results and records of calculations. Yet until now theoretical physics, with its emphasis on particles and fields, has had no place in its vocabulary for record or symbol. The absence of symbol from the vocabulary was perhaps appropriate under the outof-date picture of an experiment as producing a stream of records in which no record depends on the records produced earlier. But today's experiments involve computer-mediated feedback, in which symbols conveying theoretical calculations and symbols conveying experimental results combine to update calculations and to steer the experiment. The physical behaviors possible as targets of investigation depend on the possibilities for the transmission of symbols, so that it no longer makes sense to leave symbol and record out of the vocabulary of theoretical physics.

With regard to the application of theory to physical situations, one thinks more clearly by picturing numeric symbols expressing both calculations and measured outcomes as resident in agents' memories, e.g. as on their tapes. Thus we see the symbols of (1) as written "on tape", in sharp distinction to any physical evidence. From Sec. 2 it follows that the symbols of an explanation can never by uniquely determined by evidence, so that a written explanation depends on a logically unpredictable choice of a theorist.

Once one sees explanatory statements this way, one is free to straddle differ-

ent theoretical frameworks (rationalizing what is done anyway): parts of any big 
experiment, such as those conducted at the Large Hadron Collider, are described in essentially Newtonian terms, while other parts are described in the language of quantum theory. One is free to think in whatever mode one chooses for one or another part or aspect of an explanation. For example, in [8], we offer a quantumtheoretic explanation, indeed two of them, for the flip-flop, a device that is also conveniently explained in terms of classical circuit theory.

\subsection{Regulation and measurement of motion.}

Traditional physics presupposes coordinate systems as mathematical constructions that one relates to physical systems. Coordinate systems are defined (at least locally) by Einstein's imagined patterns of light signals propagating between imagined proper clocks. In terms of proper clocks and signals, Einstein defined the synchronization of proper clocks fixed to a non-rotating, rigid body in free fall (i.e., a Lorentz frame), and he co-defined "time" as the readings of such proper clocks, with the implication that distance from proper clock $A$ to proper clock $B$ is defined, as in radar, in terms of the duration at $A$ from the transmission of a light signal to the return of its echo from $B$. Specifically, according to Einstein's definition of the synchronization of proper clocks [18], clock $B$ is synchronous to clock $A$ if at any $A$-reading $t_{A}, A$ could send a signal reaching $B$ at $B$-reading $t_{B}$, such that an echo from $B$ would reach $A$ at $A$-reading $t_{A}^{\prime}$, satisfying the criterion

$$
t_{B}=\frac{1}{2}\left(t_{A}+t_{A}^{\prime}\right)
$$

Unlike logical synchronization with its explicit dependence on idiosyncratic responses to phases, Einstein's synchronization criteria are blackboard criteria that take no account of the responses to phases necessary to the communication of symbols. To implement a coordinate frame, actual signals conveying numerals as symbols are necessary. In [4, 5] we tell how, in a generic curved spacetime, there is no dense set of clocks that can pairwise satisfy Einstein's synchronization criterion, but a finite network of symbol-handling agents can act as a reference frame; furthermore, such a network can serve as a detector of gravitational radiation. In [4] we also note that clocks on a rotating platform (such as a merry-go-round) can never satisfy Einstein's synchronization criteria but still can be logically synchronized.

In practice, computerized signal-handling agents take part in the generation of Universal Coordinated Time (UTC), as well as in the operation of the Global Positioning System (GPS). As noted above, to generate time broadcasts, NIST must adjust the rates at which its clocks tick in relation to one another. This 
adjustment depends on the communication of numeric symbols in a system of feedback loops [5].

Besides noticing that time broadcasts depend on networks of agents that respond to unpredictable deviations, we call attention to the freedom of investigators to "build their own time", tailored to their particular investigations. This is routine in experiments investigating the instability of cutting-edge optical clocks: one achieves much higher precision by comparing one clock directly against another; the idea of comparing each to NIST time makes no sense, for the best optical clocks have much smaller instabilities than does NIST time. Similar direct comparison that bypass "time broadcasts" are required for LIGO.

\subsection{Occurrences of outcomes used promptly in feedback.}

Quantum-theoretic models predict probabilities of outcomes but the occurrence or non-occurrence of an outcome at a particular trial of a generic measurement is, by postulate, unpredictable. In experiments without feedback, the occurrences of outcomes over a run of trials are tallied up but not otherwise acted on, a fact that can obscure the significance of the unpredictability of occurrences of outcomes. With feedback in which an agent responds promptly to one or a few occurrences of outcomes to bring about physical behavior not otherwise attainable, as discussed above, one sees essential unpredictability at work, not just in the mind of the physicist, but also on the workbench of experiments, for example in a photo-detector that may or may not respond to light at the single-photon level.

\subsection{Changes in principle brought by the recognition of symbol-handling agents.}

Timing controlled by feedback stands in contrast to "time" as a concept in physics, whether the "time" is that of Newton or that of Einstein, for both concepts of "time," stand outside of the whatever is under investigation. As Newton put it:

Absolute, true, and mathematical time, in and of itself and of its own nature, without reference to anything external, flows uniformly and by another name is called duration. Relative, apparent, and common time is any sensible and external measure (exact or nonuniform) of duration by means of motion; such a measure-for example, an hour, a day, a month, a year-is commonly used instead of true time [19].

While Newton says that "true time" does not refer to anything external, this is a fudge, because he postulates "true time" as externally provided, independent of what any person or other entity of interest does. Two centuries after Newton, Einstein made time relative to the concept of a proper clock, but the proper clock is 
imagined to tick at a rate that is again externally provided. And neither in Newtonian physics nor in special or general relativity is the distinction drawn between evidence on the laboratory bench and the formulas written on a blackboard, making it a challenge to think in terms of this distinction; yet the distinction between evidence and its explanations must be made in coming to grips with the role of symbols in physics.

\subsubsection{Experimental freedom to set aside the assumption of spacetime.}

The assumption of a spacetime manifold as an explanatory principle has had a dominant place in physics for decades; these days, however, from a number of sources, one would like to be able to set aside that assumption. An issue is that the assumption of a spacetime manifold is built into the reference system which the International Astronomical Union (IAU) offers for the location of events, such as the event of the tick of an agent's clock [20]. Although for many purposes convenient, the assumption of a spacetime manifold in an unnecessary impediment to exploration. As shown in [4], the records of a network of agents of clock readings at the transmission and the reception of signals form a basis, free of any assumption of a manifold, against which to experimentally test hypotheses of spacetime manifolds.

\subsubsection{Limitations on the interleaving of sequences.}

Consider the case of a three-way race among signals $X, Y$, and $Z$ arriving at a place at which they are to be temporally ordered. Such a comparison involves pairwise balancing, involving flip-flops or their equivalent as decision elements. Each of the three signals fans out to allow three separate pairwise comparisons of which came before which. In a close race, teetering in all three pairwise comparisons can result in finding: $X$ before $Y, Y$ before $Z$, and $Z$ before $X$, rather than the "expected" $X$ before $Z$, violating the transitivity of an ordering relation, and suggesting a limit on the validity of even local temporal ordering. Making sense out of temporal order requires distinguishing the question of which cycle a symbol recognition occurred from the question of the phase of a cycle at which a signal arrived [21].

\subsubsection{Irreversibility of unpredictable events.}

The basic equations of physics involve a time variable $t$ and are invariant under the transformation $t \rightarrow-t$. When the mathematical language of experimental physics is over-stressed, this invariance appears to impose time reversibility as 
a principle of physics, in conflict with thermodynamics. But, as we have emphasized, equations written on the blackboard are not the whole story in physics. Both because of their unpredictable choices and their action in feedback loops that respond to unpredictable occurrences of outcomes, symbol-handling agents introduce a heretofore overlooked source of irreversibility into physics, even when the equations they write on the blackboard are invariant under $t \rightarrow-t$. As we explain in [22], widening the scope of descriptions admissible to physics to include the agents and the symbols that link theory to experiments opens up a new source of time-irreversibility in physics.

\subsection{Networks of symbol-handling agents as metaphors.}

And beyond the role of symbols in time broadcasts and in investigations conducted by physicists, symbols carry information in networks other than those used by people, including networks in active matter in the living world, for example, as codes written in the nucleobases of strands of DNA molecules, or the sequences of amino-acid side chains of a protein molecule.

\section{Discussion}

The first root of the recognition of symbol-handling agents came from the proof that sharpened the separation between evidence and its explanations. Our awareness of this separation comes and goes; this awareness is a limited resource, hardly to be maintained in the midst of calculations, so one can have no "onceand-for-all" separation; however; we can and do find occasions to separate our thinking at the blackboard from our thoughts about the bench to which we apply the blackboard, and sometimes this turns out to be very productive. An example to do with cryptography is mentioned at the end of [5]. When we recognize that our connecting of an explanation to evidence takes an act of our own guesswork, we recognize our own agency in the physical world.

The recognition of our own agency, our own participation in guesswork, influenced by our own individuality, opposes a long history of efforts to claim for physics an "objectivity" that goes back to the scientific outlook of Descartes. As Riskin summarizes Descartes's stance:

Seeing the world as pure machine, lifting his thinking soul out of the world, even out of its own bodily interface with the world, Descartes accomplished the distancing of self from world that defines modern subjectivity, the sense of a fully autonomous, inner selfhood, and 
modern objectivity, the sense of regarding the world from a neutral position outside of it [23].

In Sec. 2 we reviewed the separation of the blackboard of theory from evidence on the work bench, leading to the necessity of a guess beyond the reach of logic to bridge that separation. It is a guess that selects an explanation of evidence, or even that narrows the selection to any finite number of explanations. Because explanations enter both the design and the operation of experiments that generate evidence, the unavoidable guess makes a place in physics for the personal acts of imagination of physicists.

The proven dependence of physics on guesses as acts of imagination refutes any claim of a quantum explanation to an "objectivity" that aspires to produce a "final truth" from a neutral position outside the world investigated. As an investigator, I work with guessed assumptions, some of which I change from time to time. I climb about about on a "tree of assumptions," able, perhaps, to let go of this or that assumption, but only by taking hold of other assumptions [8]; there is no way for me (or for you) to look at the tree of assumptions from outside it. Thus "objectivity" as a neutral position outside the world investigated makes no sense even as a goal, but a different, less global, notion of objectivity survives, in that, under appropriate circumstances, logical communication that two agents can agree about remains possible. By way of illustration, after the experiments on the reception of signals discussed in Sec. 4, we did some experiments on people counting. As written in notes of JMM:

In 1982, I asked my son Sam and his friend Gordon to act as agents in some experiments on counting. I put a few paper cups on an otherwise bare table and asked them to each write down on a slip of paper the number of cups. In one trial they looked at the table together, in another trial they entered the room one after another; in both cases, unsurprisingly, they counted the same number of cups. Then we did another series of trials in which the two boys viewed the same table at the same time, as told by a second hand on wall clock that they could both see. At each trial I first cleared the table and then gave the boys a starting signal some seconds before the second hand crossed the twelve o'clock mark. While they watched the table I would put paper cups on it, and in some trials I would slide some cups and remove some while adding others. Their job was to each separately write down the number of cups on the tables as the clock hand passed 12. While they would get the same number if the cups were not in motion, 
when I fiddled with the cups while they counted them, they usually wrote down counts that were in disagreement, e.g one wrote down " 8 " while the other wrote down " $11 . "$

From that experiment come two interesting results. One was bringing down to the work bench, in this case the table, the notion of an objective number as a number reported by interchangeable agents, and so independent of which boy reported it. The other was the distinction between the situation in which change was blocked while a count was made-in effect logical synchronization-and the situation which, as in the experiments on logical confusion, the moving and the viewing were unsynchronized, leading to disagreement. We find then that objective counts are indeed possible but only under circumstances of logical synchronization.

While we forbear to try to explain the empirical propensity of agents at all levels from cells to civilizations to form and operate networks of logically synchronized symbolic communication, we point to this propensity as an overlooked cosmic order, ripe for further investigation.

\section{Acknowledgment}

Our thanks to Samuel Myers and Gordon Burnes for taking part in counting experiments, and to Jessica Riskin for an exchange of emails concerning the historical pulling and tugging over the role in physics, or lack of it, for an agent. We thank Kenneth Augustyn for his many considered questions and comments. We are indebted to our anonymous reviewer for motivating us to make explicit some of our core assumptions.

\section{References}

[1] J. Canales, The Physicist and the Philosopher: Einstein, Bergson, and the Debate That Changed Our Understanding of Time (Princeton University Press, Princeton, NJ, 2015)

[2] R. P. Crease, "Critical Point: A timely matter," Physics World 29, No. 1, 19 (2016).

[3] J. M. Myers and F. H. Madjid, "Freedom of choice in tracking an atomic resonance," Proc. SPIE 8400, Quantum Information and Computation X, E. Donkor, A. R. Pirich; H. E. Brandt, Eds., p. 8400-0P (8 May 2012); doi: 10.1117/12.918868. 
[4] J. M. Myers and F. H. Madjid, "Unpredictability and the transmission of numbers," Quantum Inf Process 15,1057-1067 (2016); also at http://arxiv.org/abs/1508.01119.

[5] J. M. Myers and F. H. Madjid, "Distinguishing between evidence land its explanations in the steering of atomic clocks," Annals of Physics 350, 29-49 (2014); arXiv:1407.8020.

[6] Wikipedia contributors, "Church-Turing thesis," Wikipedia, The Free Encyclopedia, https://en.wikipedia.org/w/index.php?title=Church\%E2\%80\% 93Turing_thesis\&oldid=726833164 (accessed July 8 2016).

[7] R. Van Gulick, "Consciousness," The Stanford Encyclopedia of Philosophy (Spring 2014 Edition), Edward N. Zalta (ed.), http://plato.stanford.edu/archives/spr2014/entries/consciousness/.

[8] F. H. Madjid and J. M. Myers, "Matched detectors as definers of force," Ann. Physics 319 (2005), 251; http://arxiv.org/abs/quant-ph/0404113.

[9] J. M. Myers and F. H. Madjid, "Ambiguity in quantum-theoretical descriptions of experiments," in K. Mahdavi and D. Koslover, eds., Advances in Quantum Computation, Contemporary Mathematics Series, vol. 482 (American Mathematical Society, Providence, I, 2009), pp. 107-123; http://arxiv.org/abs/1409.5678.

[10] J. M. Myers and F. H. Madjid, "What probabilities tell about quantum systems, with application to entropy and entanglement," in A. Bokulich and G. Jaeger, eds., Quantum Information and Entanglement, Cambridge University Press, Cambridge UK, pp. 127-150 (2010); available as http://arxiv.org/pdf/1409.5100.

[11] A. M. Turing, "On computable numbers with an application to the Entscheidungsproblem," Proc. London Math. Soc., Series 2, 42, 230-265 (1936).

[12] H. Meyr and G. Ascheid, Synchronization in Digital Communications (Wiley, New York, 1990).

[13] H. Meyr, M. Moeneclaey, and S. A. Fechtel, Digital Communication Receivers: Synchronization, Channel Estimation, and Signal Processing (Wiley, New York, 1998). 
[14] C. E. Shannon, "A mathematical theory of communication," The Bell System Technical Journal, Vol. 27, pp. 379-423, 623-656, July, October, 1948.

[15] T. J. Chaney and C. E. Molnar, "Anomalous behavior of synchronizer and arbiter circuits," IEEE Trans. Computers C-22, No. 4, 421-422 (1973).

[16] H. J. Gray, Digital Computer Engineering (Prentice Hall, Englewood Cliffs, NJ, 1963), pp. 198-201.

[17] J. H. Anderson and M. G. Gouda, "A new explanation of the glitch phenomenon," Acta Informatica 28, No. 4, 297-309 (1991).

[18] A. Einstein, "Zur Elektrodynamik bewegter Körper," Annalen der Physik, 17, 891-921 (1905).

[19] I. Newton, The Principia, trans. by I. B. Cohen and A. Whitman (University of California Press, 1999).

[20] M. Soffel et al., "The IAU resolutions for astrometry, celestial mechanics, and metrology in the relativistic framework: explanatory supplement," The Astronomical Journal, 126, 2687-2706 (2003).

[21] J. M. Myers and F. H. Madjid, "Rhythms of Memory and Bits on Edge: Symbol Recognition as a Physical Phenomenon," http://arxiv.org/pdf/1106.1639 (2011).

[22] J. M. Myers and F. H. Madjid, "Irreversibility in physics stemming from unpredictable symbol-handling agents," Proc. SPIE 9873, Quantum Information and Computation IX, 98730K (19 May 2016); doi: 10.1117/12.2223942; http://arxiv.org/pdf/1604.06771.

[23] J. Riskin, The Restless Clock (University of Chicago Press, Chicago, 2016) 